\section{Docetaxel versus mitoxantrone in men with hormone-refractory prostate cancer}

In 2004, the TAX 327 study investigators reported that high-dose docetaxel given every 2 weeks produced better survival outcomes than did mitoxantrone in men with metastatic hormone-refractory prostate cancer (HRPC). In their updated analysis of this trial, Berthold et al. confirm the survival benefit conferred by docetaxel-based regimens and evaluate survival in various subgroups of patients.

The TAX 327 trial enrolled 1,006 men with progressive metastatic HRPC. All patients received prednisone $5 \mathrm{mg}$ twice daily and were randomly assigned to receive docetaxel $75 \mathrm{mg} / \mathrm{m}^{2}$ administered every 3 weeks for up to 10 cycles (D3P), docetaxel $30 \mathrm{mg} / \mathrm{m}^{2}$ administered weekly for 5 of 6 weeks for up to 5 cycles (D1P), or mitoxantrone $12 \mathrm{mg} / \mathrm{m}^{2}$ every 3 weeks; participants were followed up every 3 weeks.

This analysis found a significant difference in the median survival time of patients on the D3P regimen and those receiving mitoxantrone (19.2 months vs 16.3 months; $P=0.004$ ), which was greater than that found in the 2004 analysis (18.9 months vs 16.5 months; $P=0.009$ ). The difference in survival between patients on $\mathrm{D} 1 \mathrm{P}$ and those receiving mitoxantrone was not significant. The survival benefit conferred by D3P was maintained in patients with a greater or lesser disease burden and in the following subgroups: patients with visceral disease; those with substantial pain; individuals with a Karnofsky performance status $\leq 80 \%$; and those with PSA levels above or below $115 \mathrm{ng} / \mathrm{ml}$.

The authors conclude that high-dose docetaxel administered every 3 weeks improves survival compared with mitoxantrone in men with HRPC treated with prednisone.

Original article Berthold DR et al. (2008) Docetaxel plus prednisone or mitoxantrone plus prednisone for advanced prostate cancer: updated survival in the TAX 327 study. J Clin Oncol 26: 242-245

\section{PSA velocity does not aid long-term prediction of prostate cancer incidence}

Elevated serum PSA levels can indicate the presence of prostate cancer, although PSA levels are also elevated in some nonmalignant conditions, which affects the reliability of prostate cancer prediction. PSA levels rise sharply in patients with aggressive prostate cancer, and a recent study suggested that the rate of increase (PSA velocity) could predict lifethreatening prostate cancer $10-15$ years before diagnosis. Ulmert and colleagues evaluated data from the Malmö Preventative Medicine population-based study to compare the accuracy of a single PSA measurement versus PSA velocity in the long-term prediction of prostate cancer diagnosis.

The group analyzed archived blood samples, taken from 4,907 men (aged 33-50 years) during the period 1974-1986. Each participant supplied two samples, collected 6 years apart. In this unscreened Swedish population, the 443 prostate cancers were largely detected only when they became symptomatic. Increases in PSA levels and PSA velocity were observed in these men, up to 20 years before diagnosis. The predictive value of PSA velocity was slightly lower than that of the most recent PSA measurement (concordance 0.712 vs 0.771 ). A combined model including both PSA velocity and PSA level did not improve the accuracy of prediction.

The observed lack of additional predictive value for PSA velocity indicates that PSA levels do not increase sharply before prostate cancer diagnosis but rise gradually over many years, even in those men who present with advanced cancer.

Original article Ulmert D et al. (2008) Long-term prediction of prostate cancer: prostate-specific antigen (PSA) velocity is predictive but does not improve the predictive accuracy of a single PSA measurement 15 years or more before cancer diagnosis in a large, representative, unscreened population. JClin Oncol 26: 835-841 